\title{
Interaction of suspended cohesive sediment and turbulence
}

\author{
E.A. Toorman ${ }^{a}$, A.W. Bruens ${ }^{b}$, C. Kranenburg ${ }^{b}$ and J.C. Winterwerp ${ }^{b, c}$ \\ ${ }^{a}$ Hydraulics Laboratory, Civil Engineering Department, Katholieke Universiteit Leuven, \\ Kasteelpark Arenberg 40, B-3001 Leuven, Belgium. \\ ${ }^{b}$ Hydromechanics Section, Civil Engineering Department, Delft University of Technology, \\ PO Box 5048, NL-2600 GA Delft, the Netherlands \\ ${ }^{c}$ Delft Hydraulics, PO Box 177, NL-2600 MH Delft, the Netherlands
}

This paper describes the work done in the COSINUS project, carried out within the framework of the European MAST3 research programme, on the interaction between suspended (cohesive) sediment and turbulence, with particular emphasis on its modelling. Specific attention is given to the modelling of buoyancy damping effects and turbulence production due to internal waves. Finally, some experimental results are presented on the effect of advected turbulence to the entrainment of fluid mud.

\section{KEY WORDS}

turbulence modulation, sediment-turbulence interaction, laminarisation, internal waves, entrainment, modelling

\section{INTRODUCTION}

The presence of suspended particles in turbulent flow alters the eddy viscosity distribution over the water depth as turbulent energy is dissipated by buoyancy destruction. One of the consequences is a significant apparent bottom friction (or drag) reduction. This has important implications for the transport of cohesive sediment by flowing water, in particular for the estimation of advective transport and the entrainment rate. Sediment-turbulence interaction has been studied as part of the MAST3 "COSINUS" project with the help of numerical models using Prandtl mixing-length (PML) and $k-\varepsilon$ turbulence closures, as these presently are the turbulence models used for applied modelling of cohesive sediment transport problems. The present paper investigates how the sediment-turbulence interaction can be modelled properly. The basic approach applies to any type of suspended particles, i.e. also non-cohesive sediment. Differences occur at the level of form of the turbulence modulation correction factor, introduced in Section 3.1, which for fine (e.g. cohesive) particles cause damping (see Section 2.1).

First, a brief overview is presented of available experimental evidence and the proposed mechanisms that contribute to the modulation of turbulence. The following three sections deal with modelling aspects and strategies respectively on buoyancy damping, possible subsequent laminarisation of the flow and possible internal waves at the lutocline. In a last section before 
the conclusions, results are discussed on the entrainment of a dense layer by shear turbulence generated upstream.

\section{SEDIMENT-TURBULENCE INTERACTIONS}

Literature reviews have been carried out by Winterwerp (1999), focusing on the occurrence and behaviour of concentrated benthic suspensions, and by Toorman $(2000 \mathrm{~b})$, focusing on the modelling of sediment-turbulence interactions.

\subsection{Experimental observations}

The fact that suspended particles modify the turbulence characteristics in shear flows is known from experiments for many years. Many velocity and concentration profile data in sediment-laden flows in flume experiments can be found in the literature (e.g. Vanoni, 1946; Einstein and Chien, 1955; Lyn, 1987). With an increase in the ratio $C_{m} w_{s} / u_{*}$ (where $C_{m}$ is the depth-averaged mean concentration, a measure of the sediment load, $w_{s}$ the particle settling velocity and $u *$ the shear velocity) such flows show an increasingly significant deviation from the traditional log-velocity law for clear water. It was surmised quite early that the presence of suspended particles suppresses turbulent fluctuations and that the deviation can be accounted for by reducing the value of the von Karman parameter $\kappa$. Subsequently, Coleman (1981) made a different analysis of experimental velocity profiles. He claimed that the deviations can be accounted for by considering a wake component in the velocity profile. Correcting for this wake effect, one can keep the value of $\kappa$ constant. His analysis shows several weaknesses and has been opposed by various researchers. Further details can be found in literature reviews by Winterwerp (1999) and Toorman (2000b). The discussion on whether or not the von Karman parameter decreases with increasing stratification is still not closed. The results presented below are meant to provide some new insights.

Experimental data on direct measurement of turbulence modulation by suspended particles are scarce. Nearly all experiments are with non-cohesive particles, and the majority is restricted to pipe flows. Size dependence is observed, i.e., (near-wall) turbulence is found to be attenuated by fine particles (i.e., for particle sizes smaller than about $10 \%$ of the length scale of the energy containing eddies or the integral length scale), but enhanced by coarse particles (Gore and Crowe, 1989). Relative movement of fluid and particles has been measured by Best et al. (1997). Cellino and Graf (1999) recently published the first comprehensive data set for open-channel flow experiments with fine sand in which the fluctuations of all the velocity components and the concentration have been measured. Attempts are currently being undertaken to obtain similar data for cohesive sediments (e.g., Crapper et al., 2000; Crapper and Bruce, 2002).

\subsection{Turbulence modulation mechanisms}

Various processes are believed to contribute to the modulation of turbulent fluctuations by suspended particles (e.g. Rocabado, 1999). The most important mechanism is the damping by buoyancy forces, i.e., a mechanism in which gravity opposes upward fluctuations of the particles and, in stable stratification $(\partial \rho / \partial z<0$, with $\rho$ the suspension bulk density and $z$ the vertical distance from the bottom), downward fluctuations are hindered by higher 
concentrations of particles below. Buoyancy effects can already be significant at very low concentrations.

Furthermore, the presence of particles in a fluid increases the bulk viscosity of the mixture, which in turn enhances the viscous dissipation of turbulent kinetic energy. At high concentrations, turbulence may be dissipated by interaction between the particles, which may manifest as an additional increase in the suspension viscosity. Generally, the suspension viscosity can be semi-empirically expressed as a power law function of the concentration.

\section{MODELLING OF BUOYANCY DAMPING}

The application of the Prandtl mixing-length (PML) and the $k-\varepsilon$ turbulence models in stratified flow conditions has been studied extensively at the Katholieke Universiteit Leuven (Toorman, 1999, 2000). More complex models, such as the Reynolds stress model (e.g. Galland et al., 1997), are not considered as they do not perform any better by lack of proper calibration data in the case of sediment-laden flows (Toorman, 2000b).

\subsection{PML turbulence modelling}

The Prandtl mixing length model is based on the hypothesis that the mixing length $\ell$ in simple near-wall shear flow is proportional to the distance from the bottom. Combining this with the stress balance leads to the well-known logarithmic velocity profile. This result has been confirmed by numerous experiments. Considering the equilibrium stress balance over the entire water column in open-channel flow leads to a parabolic eddy viscosity distribution (Toorman, 2000b). However, this is only valid for homogeneous fluids.

The modelling of turbulence damping by buoyancy effects is done by modulating the clear water eddy viscosity $v_{0}$ and eddy diffusivity (or mixing coefficient) $K_{0}$ with damping factors. The momentum damping factor can be defined as $F_{m}=v_{t} / v_{0}$ (with $v_{t}$ the actual eddy viscosity) and the mixing damping function as $F_{s}=K_{s} / K_{0}$ (with $K_{s}$ the actual eddy diffusivity) (e.g. Munk and Anderson, 1948). It is generally assumed that the eddy diffusivity is proportional to the eddy viscosity, i.e., $K_{s}=v_{t} / \sigma_{s}$, where $\sigma_{s}$ is called the turbulent Schmidt number.

In order to account for the buoyancy effect, the PML has to be corrected with the damping function, i.e., $F_{m}=\ell / \ell_{0}$, with $\ell$ the actual, buoyancy-corrected mixing length and $\ell_{0}$ the mixing length in non-stratified conditions (Toorman, 2000c, 2002). Subsequently, the correct velocity gradient is written as:

$$
\frac{\partial U}{\partial z}=\frac{u_{*}}{F_{m} \kappa z}
$$

and the corresponding eddy viscosity distribution for open-channel flow is given by:

$$
v_{1}=\ell^{2}\left|\frac{\partial U}{\partial z}\right|=\left(F_{m} \ell_{0}\right)^{2}\left|\frac{\partial U}{\partial z}\right|=F_{m} \kappa u_{*} z\left(1-\frac{z}{h}\right)
$$

where $\kappa$ is the von Karman constant $(=0.41), u *$ is the shear velocity, $z$ is the distance from the bottom and $h$ is the water depth. As the basic assumptions are only valid in the vicinity of a 
wall, real eddy viscosity profiles in steady open-channel flow deviate slightly from the ideal parabolic profile, in particular in the upper half of the water column (Nezu and Nakagawa, 1993).

\section{2. $k-\varepsilon$ turbulence modelling}

As the PML model cannot account for the history of turbulence and is only valid for simple shear flows, a more complex turbulence model is preferred in applied sediment transport modelling whenever possible. At present, the $k-\varepsilon$ turbulence model seems to be the best compromise between computational cost and complexity, in particular with regard to coastal and estuarine engineering applications.

This model solves the conservation of turbulent kinetic energy $k$ :

$$
\frac{\partial k}{\partial t}+U_{j} \frac{\partial k}{\partial x_{j}}=\frac{\partial}{\partial x_{j}}\left(\left(v+\frac{v_{t}}{\sigma_{k}}\right) \frac{\partial k}{\partial x_{j}}\right)+P+G-\varepsilon
$$

and its dissipation rate $\varepsilon$.

$$
\frac{\partial \varepsilon}{\partial t}+U_{j} \frac{\partial \varepsilon}{\partial x_{j}}=\frac{\partial}{\partial x_{j}}\left(\left(v+\frac{v_{t}}{\sigma_{\varepsilon}}\right) \frac{\partial \varepsilon}{\partial x_{j}}\right)+\frac{1}{T_{t}}\left(f_{1} c_{1} P+c_{3} G-f_{2} c_{2} \varepsilon\right)
$$

where $U$ is the mean velocity, $t$ is the time, $x_{j}$ are the components of the co-ordinate vector, $v$ is the kinematic viscosity of the suspension, $v_{t}=f_{\mu} c_{\mu} k^{2} / \varepsilon$ is the eddy viscosity, $T_{t}=k / \varepsilon$ is the (high-Reynolds number) turbulence time scale, $P$ is the shear production and $G$ the buoyancy term. The last two are respectively defined as:

$$
\begin{aligned}
& P=v_{t}\left(\frac{\partial U_{i}}{\partial x_{j}}+\frac{\partial U_{j}}{\partial x_{i}}\right) \frac{\partial U_{i}}{\partial x_{j}} \\
& G=\frac{g}{\rho} \frac{\nu_{t}}{\sigma_{s}} \frac{\partial \rho}{\partial z}
\end{aligned}
$$

with $g$ the gravity constant. The remaining coefficients have been determined semi-empirically (e.g. Rodi, 1980). The value of $c_{3}$ in stable stratified shear flows is generally somewhere in the range of $0-0.3$ (Rodi, 1980). Here we will adopt $c_{3}=0$. The factors $f_{\mu}, f_{1}$ and $f_{2}$ are correction functions for the low-Reynolds number formulation (see Section 4); their value is 1 for the standard high-Reynolds number form.

This model assumes isotropic turbulence and is only valid for high-Reynolds number flows. In stable stratification by suspended sediments, i.e., increasing concentration with depth, which usually is the case in natural waters, the buoyancy term is negative, i.e., turbulence is destroyed as gravity works against the turbulent fluctuations. As the turbulent Schmidt number appears in the buoyancy term, empiricism is still required in this model. Finally, the wall boundary conditions of the $k-\varepsilon$ model are based on the assumption of equilibrium in the nearwall layer and on the PML model as the bridging function to provide the missing information 
from the wall boundary layer, which is not resolved by the $k-\varepsilon$ model as the corresponding equations are not valid at a solid boundary (e.g., Toorman, 2000c). These boundary conditions introduce further dependence on empirical damping functions, as shown in the following section.

\subsection{Determination of the buoyancy damping functions}

Traditionally, the damping functions are chosen as simple empirical functions of the gradient Richardson number $R i$, which characterises the degree of stratification, of the form $F_{m}=(1+A R i)^{-a}$ and $F_{s}=(1+B R i)^{-b}$, where $A, B, a$ and $b$ are empirical parameters, such as the well-known damping functions (for free turbulence) proposed by Munk and Anderson (1948).

A distinction needs to be made between damping functions for free turbulence and for wallturbulence. Those for wall turbulence are often expressed as a function of the Monin-Obukov length-scale $L=z / R f$ (with $R f$ the flux Richardson number) and show a stronger decrease with $R i$ than the free turbulence damping functions. The empirical coefficients are found as $-5<A$ $<-10$ and $a=-1$ (Rodi, 1980). One of the reasons for the difference in behaviour is the fact that in the neighbourhood of the bottom the development of internal waves is reduced and vertical mixing by internal wave breaking is prevented (Uittenbogaard, 1995a).

As eventually only $F_{m}$ and $\sigma_{s}$ are used in the models, it is proposed to use a similar empirical form for the turbulent Schmidt number, i.e.:

$\sigma_{s}=\sigma_{0}(1+\alpha R i)^{\beta}$

with $\sigma_{0}$ the neutral Schmidt number, empirically found to have a value of approximately 0.7 (Turner, 1973), and $\alpha$ and $\beta$ empirical constants. Various experimental data for $F_{m}$ and $\sigma_{s}$ can be found in the literature, the majority of which come from fresh-salt water experiments. When plotted as a function of $R i$, the data points show considerable scatter (Fig. 1), suggesting that a dependence on $R i$ alone is unsatisfactory. Wall-effects and horizontal gradients may partially explain the scatter. A best fit can only be proposed for individual data sets. Furthermore, one can expect that similar data for sediment stratification would show additional dependence on the ratio $w_{s} / u *$.

Kranenburg (1998) has derived theoretical conditions for the empirical constants. After correction for consistent implementation of the damping functions (Toorman, 2000b), the sufficient condition for stability leads to the following condition for the exponents:

$1+a-b \geq 0$

Equality is obtained in the case of the existence of a critical flux Richardson number $R f_{c}$ at which turbulence is completely damped (i.e., total turbulence collapse). The condition $1+a-$ $b=0$ is fulfilled for the Munk-Anderson damping functions and yields the same asymptotic behaviour for large $R i$ as the theoretically derived turbulent Schmidt number relationship by Ellison (1957), given by:

$$
\sigma_{s}=\sigma_{0} \frac{(1-R f)^{2}}{1-R f / R f_{c}}
$$


(see also Turner, 1973) and is shown in figure 1. This relation can be converted into a $3^{\text {rd }}$ degree relation between the Schmidt number and $R i$. Comparison with the various data sets suggests that the value of $R f_{c}$ is case dependent and can vary widely over an order of magnitude (Fig. 1). For $0.5<R f_{c}<0.9$ this formulation reaches a local minimum of $\sigma_{s}$, as seems to be found in some experiments (i.e. Webster, 1964; Shiono et al., 2000). These observations indicate that there must be other parameters which control the Schmidt number. Notice that the ratio $w_{s} / u_{*}$ does not play a role in these data as they are all (except the Schultz ground data) for non-buoyant stratification (i.e., $w_{s}=0$, as no particles are involved).

The condition $1+a-b=0$ implies that $\beta=1$ in eq.(7). However, the existence of a critical $R f_{c}$ for turbulence collapse causes numerical problems near the free surface in some cases. This is most clearly illustrated with a $1 D V$ case, where the free surface stress-free boundary condition reduces to $\partial U / \partial z=0$, resulting in a very large $R i$. Consequently, the damping at the free surface is over-predicted, compared to reality (wherein the physical free surface boundary conditions are more complicated), in particular for the $k-\varepsilon$ model, making it impossible to mix the sediment up to the surface at very high $u_{*}$. Therefore it is advised to take $\beta<1$, e.g. $\beta=$ 0.8 as in the proposed curve in fig.1 (Toorman, 2000c). The problem can be overcome by generalising the definition of the Richardson number to include diffusion, following Ivey and Imberger (1991) (Toorman, 2000c, 2002). The latter solution seems to be the better one, as it is physically based, but requires further research.

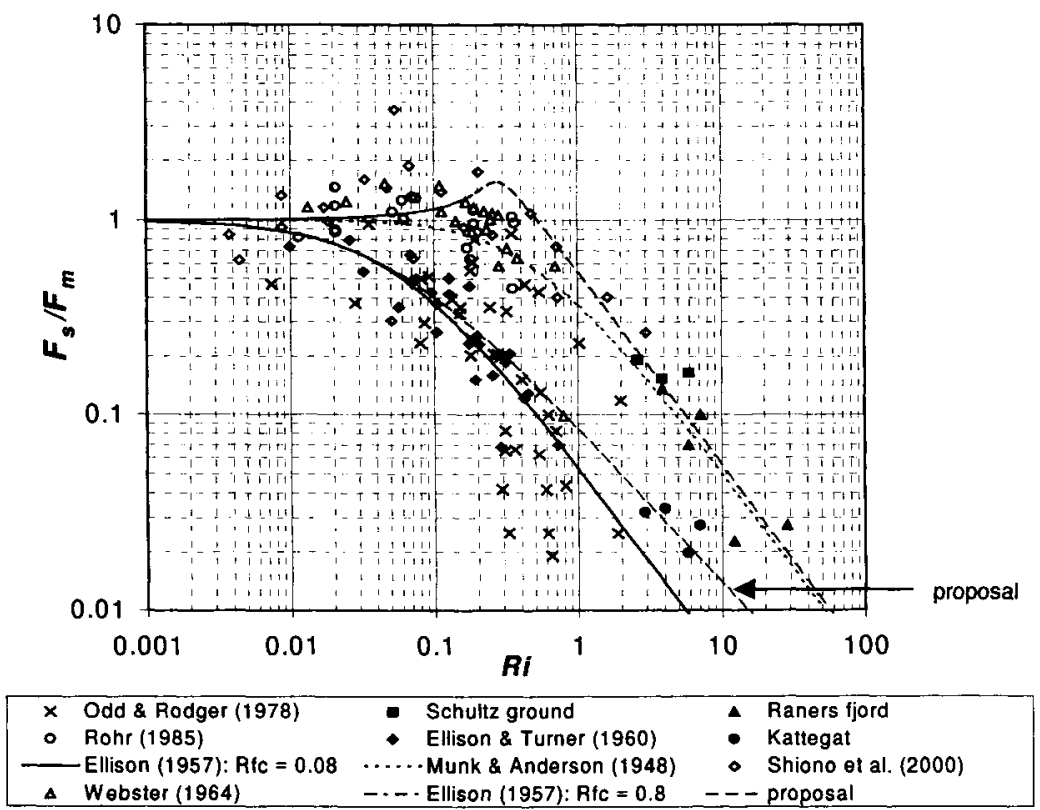

Figure 1. Experimental data for the inverse normalised Schmidt number $\sigma_{0} / \sigma_{s}$ $\left(=F_{s} / F_{m}\right)$ as a function of the gradient Richardson number and a few proposed closure relationships. Schultz ground and Raners fjord data from (Munk and Anderson, 1948); Kattegat data from (Ellison and Turner, 1960). 
The lack of accurate data is one of the major problems which prevents us from proposing a better solution. It is hoped that with the progress in computer capacities, data from numerical experiments with direct numerical simulations (DNS) of sediment-laden flows at realistic scales will become available and will help understand the trends in the experimental data and their possible dependency on other parameters.

In addition to experimental data, the solution of the $k-\varepsilon$ model can in principle also be used to determine the damping function $F_{m}$ by numerical experiments because the buoyancy effect is accounted for by the term $G$ in the $k$-equation (Toorman, 2000c). Unfortunately, the results (Toorman, 2000c) depend on the choice of the Schmidt number closure, for which no definite solution exists. Nevertheless, they suggest a linear dependence of the form $F_{m}=1-c R i$ for small $R i$ ( $R i<0.1$, with $c$ an empirical parameter), similar to the Monin-Obukov relation (Rodi, 1980). Another problem with these experiments is the deviations found near the free surface, where the boundary conditions for the $k-\varepsilon$ model are not well established; hence these numerical data are required to be discarded.

Toorman (1999) theoretically found that for the critical flux Richardson number $R f_{0}$ at which the vertical gradient $\partial R f / \partial z=0$, the momentum damping function reaches the value $F_{m}\left(R f_{0}\right)=\sigma_{s}\left(R f_{0}\right) w_{s} / \kappa \mathcal{U}_{*}$. As this value depends on the Schmidt number for the same condition, the exact value is not known.

The key to progress seems to be in finding the proper closure for the turbulent Schmidt number. The collected pieces of the puzzle presented above are still insufficient to propose a solution which provides the desired accuracy.

\subsection{Consistent bottom boundary treatment}

Traditionally, numerical models employ so-called wall functions for the determination of the conditions at solid walls, such as the bed. However, they do not account for the effect of turbulence damping. This leads to significant over-estimations of the bottom shear stress or $u *$. A simple numerical test is the verification of the shear velocity for open-channel flow driven by a constant pressure gradient, for which theoretical value is $u_{*}=\left(\rho^{-1} h \mathrm{~d} p / \mathrm{d} x\right)^{1 / 2}$. The velocity gradient as expressed by eq.(1) is used to calculate $u *$ For the $k-\varepsilon$ model, a more accurate estimation can be done using the stress balance (Toorman, 2000c, 2002). Hence it is advised that the velocity gradient in the wall node is directly estimated from the computed velocity profile in the grid cell adjacent to the wall, employing simple interpolation functions.

For the bottom boundary conditions of the momentum equations, the velocity in the nearwall node needs to be determined. Integration of eq.(1) yields the velocity profile in the wall layer, introducing an additional integral term to the logarithmic profile due to $F_{m}$. However, the damping function is generally not known as a function of $z$. It is then proposed to write the velocity profile as:

$U=\frac{u_{*}}{\kappa z} \ln \left(\frac{z}{\alpha z_{0}}\right)$

where $z_{0}$ is the roughness height of the bed and $\alpha$ the apparent roughness correction factor, which is related to the damping function by (Toorman, 2000e): 
$\frac{1}{F_{m}}=1-\frac{z}{\alpha} \frac{\partial \alpha}{\partial z}$

A series of numerical experiments (Toorman, 2000e) suggest that $\alpha$ can be parameterised as:

$$
\alpha=\exp \left(-\left(1+\beta w_{s} / u_{*}\right)\left(1-\exp \left(-b R i^{m}\right)\right)\right)
$$

with $w_{s}$ the settling velocity, $\beta, b$ and $m$ empirical constants, and $R i$ calculated at the near-wall node. Physically, the apparent change in bottom roughness corresponds to drag reduction, which has been observed both in nature and in the laboratory (Toorman, 2002).

\section{LAMINARISATION}

When density gradients are large, the damping of turbulence by buoyancy may ultimately become so strong that turbulence cannot be maintained and the flow becomes laminar. Two situations, illustrated by the numerical example shown in figure 2, need to be considered.

Generally, high-density peaks occur at the bottom due to sedimentation and the presence of the viscous sublayer. Turbulence damping occurs along with drag reduction and the subsequent thickening of the viscous sublayer, compared to the clear water case. Toorman (2000e) has shown that the consistent boundary treatment method leads to drag reduction predictions of the same order of magnitude as measured by Li and Gust (2000).

The second possible situation is the generation of a lutocline as the result of the combined effect of hindered settling and buoyancy damping in turbulent shear flows. The occurrence of such two-layer sediment-stratified systems in some estuaries has been observed by Wolanski et al. (1988). It should be realised that such two-layer systems cannot be simulated correctly by the PML model, because the lutocline forms a new reference for calculation of the mixing
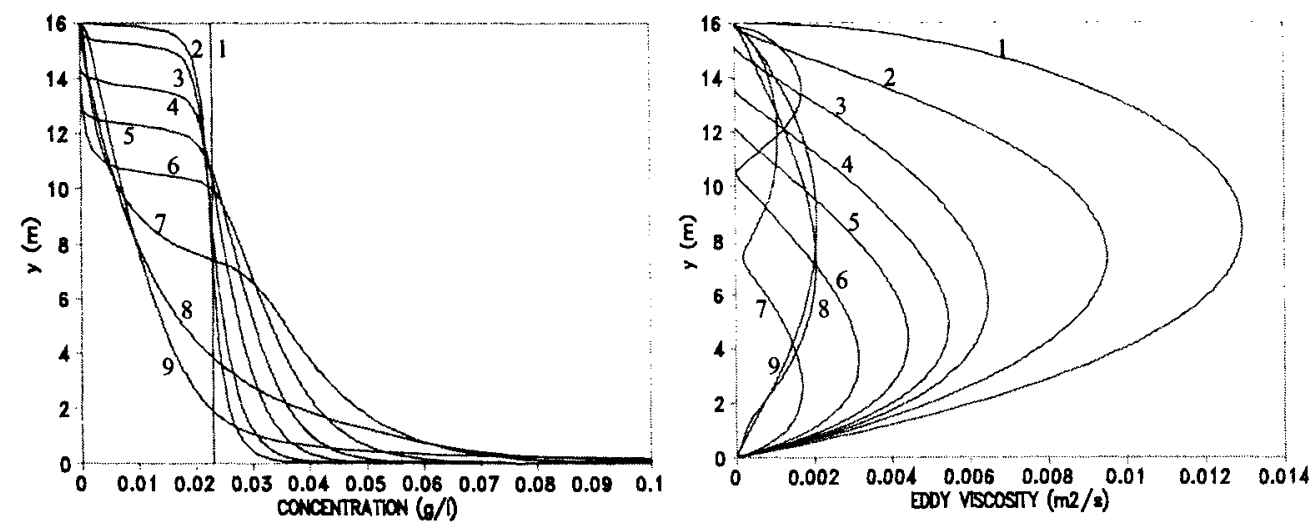

Figure 2. Numerical results ( $k-\varepsilon$ model) of the evolution $(1 \Rightarrow 9)$ of concentration and corresponding eddy viscosity profiles of an initially homogeneous suspension $\left(\mathrm{C}_{0}=23 \mathrm{mg} / \mathrm{l}\right)$ at a constant flow rate (mean flow velocity $\left.=0.2 \mathrm{~m} / \mathrm{s}\right)$, using a constant turbulent Schmidt number $\left(\sigma_{s}=\sigma_{0}=0.7\right)$. 
length in the upper layer. The second to the fifth eddy viscosity profiles (from left to right) in figure 2 show that turbulence may be completely damped in the upper, sediment-free layer. Whether complete laminarisation really occurs above lutoclines has never been demonstrated experimentally. It is very likely that the density interface becomes unstable, resulting in internal waves, which may increase mixing and turbulence production (see next section). This effect is not included in the model used to obtain the results of fig. 2 .

Thus far, the modelling of laminarisation has only been successful for relatively simple shear flows where it occurs along the wall, as in the first case. In actuality, because cohesive sediment transport problems are time-dependent, they involve variations of stratification and hence the thickness of the viscous sublayer. In general (in the absence of fluid mud) this thickness will remain much smaller than the vertical grid size, but problems may occur around flow reversal at slack and neap tides, when the sublayer thickness may become very large and fluid mud layers may form. Otherwise, as long as the sublayer thickness remains small, the relevance of modelling near-wall laminarisation may be reduced in commercial models, as they can handle flow reversal due to the fact that a constant horizontal diffusion is used. This is so because the horizontal grid scales are too large compared to the turbulence length scales of the PML or the $k-\varepsilon$ model.

In principle, the sublayer requires a different method of solution than the fully turbulent layer, because the assumptions for the above mentioned turbulence models are no longer valid. In the PML model for clear water, investigated at the T.U.Delft (Kranenburg, 1999), a twolayer approach can be applied, where in the near-wall layer another mixing length model, i.e., a modified Van Driest model, is used:

$$
\ell(z)=\ell_{0}(z)\left[1-\exp \left(-\frac{1}{A} \frac{u_{*} z}{v}\right)\right]
$$

where the neutral mixing length distribution for free-surface flow is given by:

$$
\ell_{0}(z)=\frac{\kappa z \sqrt{1-z / h}}{1+\Pi(\pi z / h) \sin (\pi z / h)}
$$

with $\Pi=$ Coles' wake strength parameter (Nezu and Rodi, 1986). The interface with the fully turbulent layer is then determined by a new laminarisation criterion:

$$
\frac{v_{t m}}{v}<c \sigma_{0} \operatorname{Re}_{t c}
$$

The selected value of the critical turbulent Reynolds number $R e_{t c}$ is 15 and $c=0.61$, as obtained by a numerical calibration procedure. The method has been successfully validated with the simulation of the laminarising duct flow of a homogeneous fluid. The model has also been applied to slowly decelerating, sediment-laden, open-channel flows. The mixing length model (eq.12) then has to be multiplied with the factor $F_{m}$ to account for turbulence modulation by suspended particles ( $c f$. Section 3.1).

In the $k-\varepsilon$ model the problem can, in principle, be handled by correcting various constants with near-wall damping functions $\left(f_{\mu}, f_{1}\right.$ and $\left.f_{2}\right)$, which is known as a low-Reynolds number 
turbulence model. This has been investigated at the K.U.Leuven. Toorman (2000d) proposed a new realisable time scale (i.e. a time scale which adapts itself from the laminar flow Kolmogorov scales to the $k-\varepsilon$ scales, depending on the turbulent Reynolds number), which defines the damping function $f_{2}$. His analysis of direct numerical simulation (DNS) data furthermore indicates that the coefficients $\sigma_{k}$ and $\sigma_{\varepsilon}$ cannot have a constant value within the near-wall layer. Again, however, the damping functions proposed in the literature are only valid for homogeneous fluids and nearly all of them are restricted to smooth walls (Rocabado, 1999). Furthermore, this model requires a very fine grid size near the bottom, which considerably increases the computational cost.

Alternatively, the $k-\varepsilon$ model can also be combined with a wall layer model, where a VanDriest type mixing-length model is used. This is known as the two-layer approach (Toorman, 2000d). A Reynolds number criterion is used to determine the boundary between the two layers. The same problems as for the two-layer PML model apply. Unfortunately, the accuracy of the two-layer approach is generally not very high for coarse grids.

None of the above methods can be applied to laminarisation away from the wall, such as may occur around lutoclines. The so-called "wall-distance free" low-Reynolds number models have recently been proposed in the literature, but they are complicated and are meant for nearwall turbulent shear flows (Toorman, 2000d). Nevertheless, presently used codes seem to be able to handle laminarisation around a lutocline, as figure 2 proves. As the molecular viscosity is too small to stabilise the solution, this is only possible due to numerical diffusion inherent to the numerical schemes, especially in commercially applied codes which require robustness, or to specially designed locally added artificial diffusion in the $k-\varepsilon$ turbulence model to stabilise the solution where the actual equations are no longer valid (Toorman, 1999). It is not known how these artificial solutions affect the history of simulated turbulence. Considering furthermore all the uncertainties due to required simplifications following the relatively coarse scales in 3D estuarine modelling, which do not capture sharp lutoclines anyhow, laminarisation away from the wall is not expected occur in applied modelling practice. Furthermore, mixing due to internal waves additionally reduces the chance of laminarisation.

\section{INTERNAL WAVES}

Internal waves do occur in stratified flows, and are most manifest at the interface of a twolayer flow system. Internal waves contribute to the vertical transport of momentum, but not directly to the transport of mass (sediment in our case). Uittenbogaard (1995a, 1995b) showed that, although internal wave breaking is essential to initiate turbulence at an interface, it is the shearing induced by the internal waves that supplies most of the energy to the turbulence. Hence, enhanced turbulence also affects vertical mass transport.

The effect of internal waves can be incorporated through additional production and dissipation terms in the $k-\varepsilon$ turbulence model. In a study by Delft Hydraulics (Winterwerp and Uittenbogaard, 1999), a more parameterised approach is followed in which the effect of internal waves is incorporated in the eddy viscosity (but not in the eddy diffusivity). 


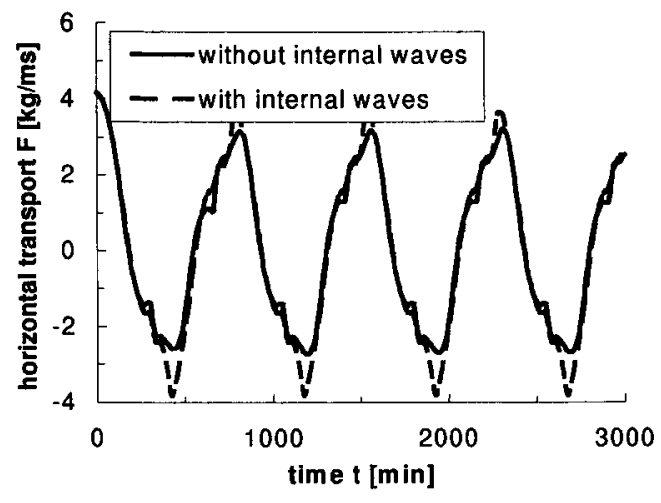

Figure 3. Variation of computed horizontal transport with time.

The effect of turbulence produced by internal waves on the dynamics of concentrated benthic suspensions (CBS) was studied through a one-dimensional approach. The 1DV momentum equation, including the effect of internal waves, reads:

$\frac{\partial u}{\partial t}+\frac{1}{\rho} \frac{\partial p}{\partial x}=\frac{\partial}{\partial z}\left[\left(v+v_{t}+v_{I W E}\right) \frac{\partial u}{\partial z}\right]$

where $u$ is the horizontal flow velocity, $p$ is pressure, $x$ and $z$ are the horizontal and vertical coordinate and $v_{I W E}$ the additional viscosity induced by internal waves.

The additional internal wave dissipation term is parameterised as a function of the buoyancy frequency, the vertical shear rate and a length scale which is related to the Ozmidov (length) scale. This additional viscosity is only relevant in case of large stratification.

This approach was implemented in a IDV point model (Winterwerp, 1999). The model was run without (as reference condition) and with internal wave effects for a hypothetical open channel flow with a depth of $8 \mathrm{~m}$, a depth-mean velocity of $0.7 \mathrm{~m} / \mathrm{s}$ and a depth-mean suspended sediment concentration of $0.74 \mathrm{~g} / \mathrm{l}$. These conditions are near saturation, hence a strong buoyancy-induced interaction exists between the turbulent flow field and the suspended sediment concentration. As a result the eddy viscosity for the reference case is considerably decreased. The horizontal sediment flux is then particularly affected at larger flow velocities, as the transport is relatively large. The net horizontal transport is defined as $F=\int_{0}^{h} u c \mathrm{~d} z$ and the results are presented in fig. 3, with and without internal wave effects. Figure 3 shows differences of about $20 \%$ during maximal velocities, whereas during the rest of the tide the differences are comparatively small. It must be noted that the asymmetric curve at flood and ebb velocity is due to plotting resolution.

From the above simulations it is concluded that the effect of augmented eddy viscosity by internal waves can be considerable for the hydrodynamic conditions examined in this paper. However, it is recommended not to include these effects on a routine basis before turbulent 
modelling of concentrated benthic suspensions is better understood, as, for instance, low Reynolds number effects may be of greater importance.

For more details the reader is referred to Uittenbogaard (1995a, 1995b) and Winterwerp and Uittenbogaard (1999).

\section{DECAY OF NON-LOCALLY PRODUCED TURBULENCE DUE TO DENSITY STRATIFICATION}

\subsection{Problem definition}

In the deeper parts of an estuary, for example in a navigation channel, the deposition rate of cohesive sediment may be high, especially during slack water, forming a CBS, which may behave as a dense fluid for several hours, or longer in the case of stirring by wave action or by passing ships. In the flow upstream of this depression, turbulence is produced mainly as a result of bed friction. In the depression, the production of turbulence is for the larger part suppressed due to the presence of sediment-induced density stratification. In turn, turbulence produced over the rigid bed upstream of the deeper part is advected over the CBS and gradually decays in the downstream direction. While decaying, this non-locally produced turbulence might entrain material from the CBS. This interaction of turbulence and density stratification has been studied at Delft University of Technology. The study consisted of laboratory experiments on the decay of non-locally produced turbulence over a CBS and the subsequent entrainment, as well as of numerical simulations of these processes. The objectives of the experiments were to obtain a relation between hydrodynamic parameters, decay of turbulence and entrainment rates, and secondly, to generate data to validate computer models. This study is summarized next.

\subsection{Scaling laws}

To scale field conditions based on laboratory experiments, several scaling laws have to be taken into account. The first scaling law is concerned with the turbulence structure. It is required that the Reynolds number $(R e=u h / v$, where $u$ is mean velocity, $h$ is depth of the flow and $v$ is viscosity) in the physical model be sufficiently high. The upstream bed is roughened to obtain hydraulically rough conditions.

The second law is concerned with the scaling for turbulence decay. A lower bound of the length scale $L$ of decay can be derived from a balance of the advection and dissipation terms in the transport equation for turbulent kinetic energy. $L$ scales with the water depth $(L \approx 9 h)$, and for significant decay to be observable, the length of the depression should be at least several times $L$.

The third scaling law concerns consolidation time. The consolidation time $T_{c}$ is proportional to the inverse of the squared depth $\delta$ of the CBS layer (i.e., $T_{c} \propto \delta^{-2}$ ). This means that in the physical model it is nearly impossible to keep the stationary CBS in a fluidised state. A mud layer, possessing strength (behaving as a non-ideal Bingham fluid when sheared), will be formed and the turbulence production at the water/mud interface then would not be essentially different from that over the upstream rigid bed.

The last scaling law deals with entrainment. The entrainment process is governed by the overall Richardson number 
$R i_{*}=\frac{\Delta \rho g h}{\rho u_{*}^{2}}$

where $\Delta \rho$ is the excess density of the CBS, $\rho$ the density of the overlying water, $g$ is the acceleration of gravity, $h$ the height of the water layer and $u *$ the friction velocity at the upstream rigid bed. Simulation of the entrainment process in a hydraulic model requires scaling at constant $R i *$, which for constant $g$ and $\rho$ yields:

$\frac{\Delta \rho_{m} g h_{m}}{u_{*_{m}}^{2}}=\frac{\Delta \rho_{f} g h_{f}}{u_{*}^{2}}$

where subscript $f$ refers to the field and $m$ to the physical model. Substituting realistic values for the field parameters, it can be shown that extremely low bed shear stresses in the physical model are required. These values are likely to be lower than the yield stress of a concentrated layer in a laboratory flume.

Based on the last two scaling laws, the CBS in the physical model is replaced by salt water. In earlier work it was already shown that in terms of initial entrainment rate, CBS behaves similar to saline water.

\subsection{Experimental set-up and results}

Two series of experiments were carried out. The first series was concerned with decay of turbulence and the second with entrainment. The tests were conducted in a flume of $30 \mathrm{~m}$ length, $1 \mathrm{~m}$ width and $0.3 \mathrm{~m}$ depth. A longitudinal cross-section of the experimental set-up for the first series is shown in Figure 4. The upstream part of the flume was $0.15 \mathrm{~m}$ deep, the deeper part was $0.3 \mathrm{~m}$ deep. To prevent flow separation the slope is small ( $\left.8^{\mathrm{o}}\right)$. The depth of the saline water in the depression was kept constant $(0.1 \mathrm{~m})$ by a continuous inflow (at a small rate) at the upstream slope and a (internal) weir near the end of the depression. After the flow became stationary, turbulent velocities were measured in the upper fresh water layer using laser-Doppler velocimetry. Figure 4 indicates the six positions (with a spacing of $2 \mathrm{~m}$ ) at which vertical velocity profiles were measured.

The entrainment rate decreased in downstream direction over the depression. To measure the integral entrainment at different distances downstream of the slope, the experimental setup was slightly changed for the second experimental series by replacing the internal weir by an internal barrier, which could be placed at various positions (for example position 2 to 6 in fig. 4). For each position the rate at which saline water had to be supplied to the flume to keep the height of the saline water layer at $0.1 \mathrm{~m}$ was measured.

The experimental program was set up to vary the overall Richardson number (by varying $\Delta \rho_{m}$ and/or $\left.u_{*}\right)$. In figure 5 the non-dimensional turbulent kinetic energy $\left(k / u^{2}\right)$ is plotted against the distance downstream of the ramp for all experiments. The decrease in entrainment rate was more or less proportional to this decay in turbulent kinetic energy. A more detailed analysis of the experimental data can be found in (Wissmann and Bruens, 2000).

\subsection{Numerical simulations}

A numerical flow model for shallow-water flow (Delft3D) with a standard $k$ - $\varepsilon$ turbulence model was used for the numerical simulation of the physical experiments. By comparison with measurements, turbulence decay predicted by the model as well as the predicted entrainment 


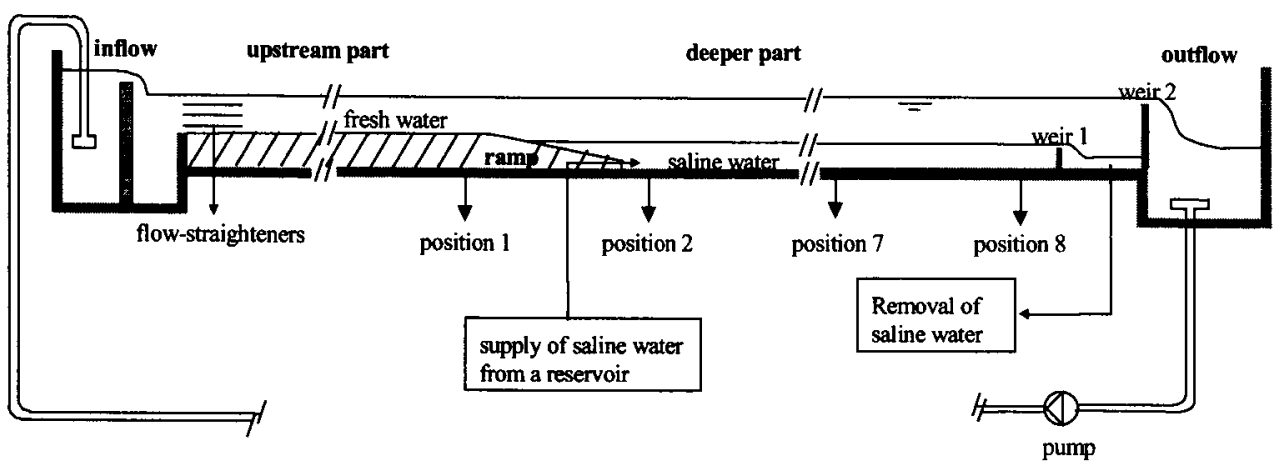

Figure 4. Schematic cross-section of the experimental set-up for the first series of experiments (not to scale).

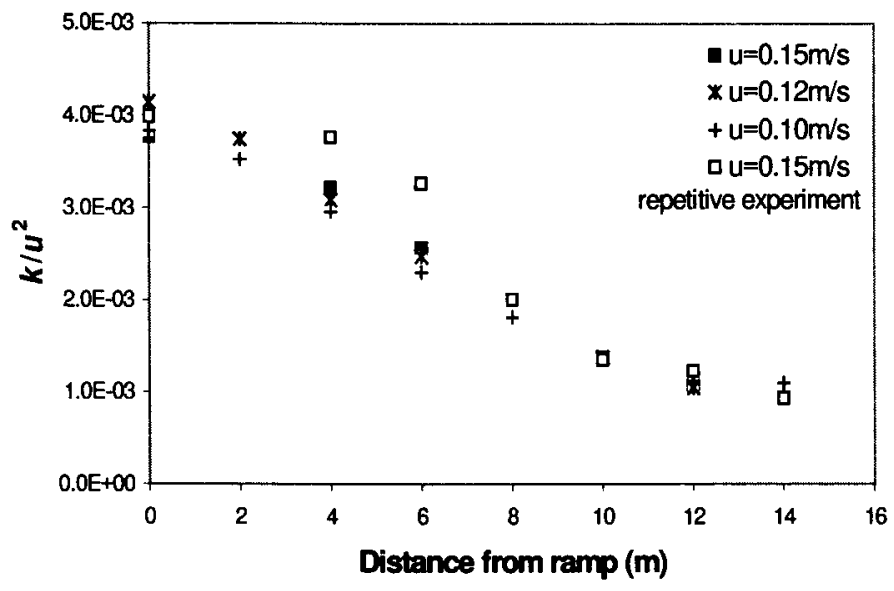

Figure 5. Measured decay of turbulent kinetic energy with distance from the ramp.

of saline water were tested. The model simulated the decay in the upper layer accurately, but the entrainment rate was underpredicted due to the fact that in the model internal waves were not taken into account.

\section{CONCLUSIONS}

Suspended cohesive sediments cause damping of the turbulent fluctuations in flowing water and alter the apparent bed roughness. Consistent implementation of buoyancy-induced turbulence damping functions allows the modelling of the damping and the bed roughness modification (or drag reduction) and can explain the decrease of the von Karman parameter 
with increasing stratification. Finding the appropriate turbulent Schmidt number closure seems to be the key to make further progress. A major problem with the further development of a validated modelling strategy for sediment-turbulence interaction in cohesive sediment transport modelling is the lack of data for testing theories, developing and calibrating more accurate damping functions and validating the models. Laboratory experiments at high enough concentrations with (cohesive) sediments require new non-optical (e.g., acoustic) measurement techniques, which are being developed and improved. Another hope is that future direct numerical simulation (DNS) data at realistic scales will become available, once computer power allows it. As long as these validation data are not available, the framework proposed in this paper provides the best possible approximation to be implemented in cohesive sediment transport models.

Turbulence damping by buoyancy can become so strong that the flow laminarises locally, i.e., near the bottom and around a lutocline. At the bottom the viscous sublayer thickens with increasing stratification. When its thickness tends to reach a value on the order of the vertical grid size of the model, it seems advisable that a more comprehensive two-layer approach should be implemented. A more systematic study is required for the numerical implementation of this phenomenon on a realistic coarse grid as used in real applications in order to evaluate the feasibility of this method.

No concern is presently required regarding possible laminarisation around lutoclines, because available models do not capture the gradients accurately enough to lead to the problem. The shortcoming, resulting in an excessive vertical diffusion, is partially compensated by the likely occurrence of internal waves, which increases vertical mixing, which is presently absent in models.

It is recognised that internal waves may become important when the degree of stratification is high. They are an additional source of turbulence production, which is missing in the presently used models. This can explain the underestimation of entrainment in certain simulations. The problem can be handled by the introduction of an additional empirical diffusion coefficient, which is only a rough parameterisation of the complex process. However, in view of the many uncertainties regarding turbulence modelling when sediment is in suspension and the limitations due to the relatively coarse grids for coastal and estuarine applications, it is advised not to implement internal wave corrections at present.

An experimental study on the effect of non-locally produced turbulence on the entrainment of a stationary, localised CBS layer (for example in a depression or a navigation channel) has been carried out. No or only a minor degree of turbulence is generated in the pool as a result of a stable interface. Turbulence produced over the rigid bed upstream of the depression is advected over the depression and decays in the downstream direction. This decay has been measured in a physical model. While decaying it entrains material from the dense layer in the depression. Preliminary results indicate that non-locally produced turbulence can entrain a substantial amount of cohesive sediment. The data obtained have been used to validate a numerical flow model which accurately simulates the decay in the upper layer.

Acknowledgements: This work has been carried out as part of the MAST3 project "COSINUS", partially funded by the European Commission, Directorate General XII for Science, Research and Development under contract no. MAS3-CT97-0082. The first author's post-doctoral position was financed by the Flemish Fund for Scientific Research. 


\section{REFERENCES}

Best, J., Bennett, S., Bridge, J. and Leeder, M. , 1997, Turbulence modulation and particle velocities over flat sand beds at low transport rates. ASCE J. Hydr. Eng., 123(12), 1118-1129.

Cellino, M. and Graf, W.H. , 1999). Sediment-laden flow in open channels under noncapacity and capacity conditions. ASCE J. Hydr. Eng., 125(5), 456-462.

Coleman, N.L., 1981, Velocity profiles with suspended sediment, J. Hydraulic Research, 19(3), 211-229.

Crapper, M., Bruce, T. and Gouble, C. , 2000, Flow field visualisation of sediment-laden flow using ultrasonic imaging, Dynamics of Atmospheres and Oceans, 31, 233-245.

Crapper, M. and Bruce, T. , 2002). Measurement of mud transport processes using ultrasonic imaging, Proc. INTERCOH-2000, J.C. Winterwerp and C. Kranenburg eds., Elsevier, this volume.

Einstein, H.A. and Chien, N. , 1955, Effects of heavy sediment concentration near the bed on velocity and sediment distribution, M.R.D. Sediment Series, No.8, Missouri River Div., US Army Corps of Engineers.

Ellison, T.H. , 1957, Turbulent transport of heat and momentum from an infinite rough plane, $J$. Fluid Mechanics, 2, 456-466.

Ellison, T.H. and Turner, J.S. , 1960, Mixing of dense fluid in a turbulent pipe flow, J. Fluid Mechanics, 8, 514-544.

Galland, J.-C., Laurence, D. and Teisson, C. , 1997, Simulating turbulent vertical exchange of mud with a Reynolds stress model, In: Cohesive Sediments, N. Burt, R. Parker and J. Watts, eds., J. Wiley, Chichester, 439-448.

Gore, R.A. and Crowe, C.T. , 1989, Effect of particle size on modulating turbulence intensity, J. Multiphase Flow, 15, 279-285.

Ivey, G.N. and Imberger, J. , 1991, On the nature of turbulence in a stratified fluid. Part I: The energetics of mixing, $J$. Physical Oceanography, 21, 650-658.

Kranenburg, C. , 1998, Saturation concentrations of suspended fine sediment. Computations with the Prandtl mixing-length model, Report No.5-98, Faculty of Civil Engineering and Geosciences, Delft University of Technology.

Kranenburg, C. , 1999, Laminarisation in flows of concentrated benthic suspensions. Computations with a low-Reynolds mixing-length model, Report No.1-99, Faculty of Civil Engineering and Geosciences, Delft University of Technology.

Li, M.Z. and Gust, G. , 2000, Boundary layer dynamics and drag reduction in flows of high cohesive sediment suspensions, Sedimentology, 47, 71-86.

Lyn, D.A. , 1987, Turbulence and turbulent transport in sediment-laden open-channel flows, PhD thesis, California Institute of Technology, Passadena, CA.

Munk, W.H. and Anderson, E.A., 1948, Notes on a theory of the thermocline, J. Marine Research, 3(1), 276-295.

Nezu, I. and Nakagawa, H. , 1993, Turbulence in open-channel flow, IAHR Monograph Series, Balkema, Rotterdam.

Nezu, I. and Rodi, W. , 1986, Open-channel flow measurements with a laser Doppler anemometer, J. Hydr. Eng., 112, 335-355.

Odd, N.V.M. and Rodger, J.G. , 1978, Vertical mixing in stratified tidal flows, ASCE J. Hydraulics Div., 104(3), 337-351.

Rocabado, O.I. , 1999, Modelling highly concentrated turbulent flows with non-cohesive sediments, PhD thesis, Civil Eng. Dept., Katholieke Universiteit Leuven. 
Rodi, W. , 1980, Turbulence models and their application in hydraulics, State-of-the-art Paper, IAHR, Delft.

Rohr, J.J. , 1985, An experimental study of evolving turbulence in uniform shear flows with and without stable stratification, $\mathrm{PhD}$ thesis, Dept. of Applied Mechanics and Engineering Sciences, University of San Diego, CA.

Shiono, K., Siqueira, R.N. and Feng, T. , 2000, Exchange coefficients for stratified flow in open channel, Proc. $5^{\text {th }}$ Int. Symp. on Stratified Flows (G.A Lawrence, R. Pieters and N. Yonemitsu, eds.), 2, 927-932, Dept. of Civil Engineering, University of British Columbia, Vancouver.

Toorman, E.A. , 1999, Numerical simulation of turbulence damping in sediment-laden flow. Part 1. The Siltman testcase and the concept of saturation, Report HYD/ET99.2, Hydraulics Laboratory, Katholieke Universiteit Leuven.

Toorman, E.A. , 2000a, Stratification in fine-grained sediment-laden turbulent flow, Proc. $5^{\text {th }}$ Int. Symp. on Stratified Flows (G.A Lawrence, R. Pieters and N. Yonemitsu, eds.), 2, 945950, Dept. of Civil Eng., University of British Columbia, Vancouver.

Toorman, E.A. , 2000b, Sediment-laden turbulent flow: a review, Report HYD/ET/00.1, Hydraulics Laboratory, Katholieke Universiteit Leuven.

Toorman, E.A. , 2000c, Parameterisation of turbulence damping in sediment-laden flows, Report HYD/ET/00/COSINUS3, Hydraulics Laboratory, Katholieke Universiteit Leuven.

Toorman, E.A. , 2000d, Analysis of near-wall turbulence modelling with the $k-\varepsilon$ turbulence model, Report HYD/ET/00/COSINUS2, Hydraulics Laboratory, Katholieke Universiteit Leuven.

Toorman, E.A. , 2000e, Drag reduction in sediment-laden turbulent flow, Report HYD/ET/00/COSINUS5, Hydraulics Laboratory, Katholieke Universiteit Leuven.

Toorman, E.A. , 2002, Modelling of turbulent flow with suspended cohesive sediment, Proc. INTERCOH-2000, J.C. Winterwerp and C. Kranenburg eds., Elsevier, this volume.

Turner, J.S. , 1973, Buoyancy effects in fluids, Cambridge University Press.

Uittenbogaard, R.E. , 1995a, The importance of internal waves for mixing in a stratified estuarine tidal flow, $\mathrm{PhD}$ thesis, Delft University of Technology, September 1995.

Uittenbogaard, R.E. , 1995b, Observations and analysis of random internal waves and the state of turbulence, Proc. IUTAM Symp. on Physical Limnology (Broome, Western Australia, September 1995).

Vanoni, V.A., 1946, Transportation of suspended sediment by water, Trans. ASCE, 111, 67133.

Webster, C.A.G. , 1964, An experimental study of turbulence in a density stratified shear flow, J. Fluid Mech., 19, 221-245.

Winterwerp, J.C. , 1999, On the dynamics of high-concentrated mud suspensions, $P h D$ thesis, Delft University of Technology, Delft.

Winterwerp, J.C. and Uittenbogaard, R.E. , 1999, Effect of internal waves on the saturation of high-concentrated mud suspensions, WL/Delft Hydraulics / Delft University of Technology, Report Z2386.

Wissmann, J. and Bruens, A. W. , 2000, Experiments on the decay of turbulence due to density stratification, Report No.6-00, Faculty of Civil Engineering and Geosciences, Delft University of Technology.

Wolanski, E., Chappell, J., Ridd, P. and Vertessy, R. , 1988, Fluidization of mud in estuaries, J. Geophysical Research, 93(C3), 2351-2361. 